\title{
Mapping and Quantifying Spatial and Temporal Dynamics and Bundles of Travel Flows of Residents Visiting Urban Parks
}

\author{
Wenping Liu ${ }^{1,2, *}$ (D), Chenlu Dong ${ }^{1}$ and Weijuan Chen ${ }^{1}$ \\ 1 Department of Landscape Architecture, College of Horticulture \& Forestry Sciences, \\ Huazhong Agricultural University, Wuhan 430070, China; qydong21@163.com (C.D.); \\ chenwj0811@126.com (W.C.) \\ 2 Key Laboratory of Urban Agriculture in Central China (pilot run), Ministry of Agriculture, \\ Wuhan 430070, China \\ * Correspondence: liuwenping@mail.hzau.edu.cn
}

Received: 28 May 2017; Accepted: 21 July 2017; Published: 25 July 2017

\begin{abstract}
Understanding the spatial and temporal movement patterns of residents visiting urban parks is essential to link park use with human well-being. There has been increasing interest recently in quantifying travel behaviors, but most studies have focused on flows of commuters within cities or tourists flows between cities. Actual, specific spatial and temporal connections between urban parks and residents within cities are still poorly understood. Here, we present an approach to take different types of travel flows into account when mapping and quantifying the spatial delivery and temporal dynamics of specific travel flows. This approach is based on empirical investigation of individuals' movements from their homes to parks and online map navigation technology. The flow maps we generated capture key travel flow patterns, addressing where, which, when, and how much they were associated with the use of recreational service provided by urban parks in Wuhan, China, during the summers of 2015 and 2016. Such maps may aid efforts to restore or improve the efficiency of park access and use. We also examined bundles of these spatial and temporal flows, and show that similar transport conditions were important for bundling spatial flows, while temporal flow bundles were significantly related to spatial features. These findings could help to reveal potential trade-offs and synergies of travel flows associated with space and time constraints and facilitate exploration of policy alternatives for equitable and efficient use of urban park systems.
\end{abstract}

Keywords: urban park; travel flow; recreational service use; spatial pattern; temporal dynamics

\section{Introduction}

Urban parks are specifically intended and designed to offer diverse recreational service, which are particularly important for enhancing the health and well-being of urban residents [1,2]. The use of the recreational services provided by urban parks largely depends on the movement of visitors from their homes to the parks [3]. Thus, the accurate description of park visitors' travel flows could help us to understand the actual spatial and temporal connections between parks and their beneficiaries. However, with the rapid development of cities, quantifying actual, specific travel flows to urban parks has become an increasingly complex task and particularly challenging in the face of unprecedented transportation networks, variations in individuals' travel choices and preferences for particular experiences [4]. Expanding knowledge of the travel flows of residents visiting urban parks is important for recreation planners and managers wishing to improve the efficient use of urban parks.

Previous research has quantified several spatial characteristics of the travel flows of urban residents using data from surveys and questionnaires [4,5]. For example, Roorda and Ruiz [6] analyzed 
the number and nature of travel flows by mode of transport, based on data from the Toronto travel activity panel survey in Canada, while Thøgersen [7] reported travel route dependent behaviors with respect to choice of public transport using data from a Danish panel survey. Some studies have analyzed travelers' attitudes towards alternative transportation, in order to provide information for urban planning and management $[8,9]$. In addition, temporal variations in travel flows have been examined in recent studies, including differences in the behavior of residents between weekdays and weekends [10] as well as seasonal variations [11]. The majority of these studies typically focus on commuting, while travel for recreational purpose has been largely ignored, although lack of knowledge of these dynamics hinders understanding the interactions between urban parks and residents. Recently, spatial characteristics of tourist flows in a city, in particular international visitor flows, have been quantified in several case studies [12,13]. Despite all this, the travel patterns of city dwellers visiting urban parks has still rarely been examined. Furthermore, limited attention has been paid to the overall characteristics of travel flows, such as flow paths, velocities, directions, times, and quantities. There is a need to examine travel flows of residents visiting urban parks in greater detail to understand the spatial and temporal connections between parks and residents.

With recent developments in mobile location technology, it is possible to track and record more information on individual travel flows. New technologies to obtain spatial-temporal travel data, such as GPS tracking [14] and smart card recording technology [15], have been applied in recent studies of travel behavior. For example, Smallwood et al. [16] explored visitor movement patterns using travel networks in a large marine park in north-western Australia, while Orellana et al. [17] revealed movement patterns of visitors in the Dwingelderveld National Park in the Netherlands using GPS technology. These new technologies have proven to be more accurate in collecting spatial and temporal travel data compared to surveys. However, most of the data collected using these new technologies cannot be directly linked to personal motivations. It is difficult to differentiate the motivation of recreational service use from any other travel purposes. Past studies have used various algorithms to compute scenic routes in order to identify recreational service flows within scenic spots, for example using Path Attribution Networks (SPANs) [18,19], a single objective shortest path algorithm [20] or road segment-based clustering of geo-tagged photos and Flickr images [21,22]. Despite these studies having the clear purpose of examining recreational service use, and recreational service flows within scenic spots being clearly quantified, the spatial and temporal connections between scenic spots and residences still remain unclear. Moreover, not all visitors use these new technologies, and the representativeness of the results of these studies is limited. There is a need to combine traditional surveys and new technology in order to identify, comprehensively, the spatial and temporal characteristics of travel flows to urban parks.

Due to substantial spatial and temporal overlaps of individual travel flows, there is a view that travel convenience, time and experience constraints restrict the set of spatial-temporal opportunities for travelling to urban parks [23]. By examining spatial and temporal bundles of travel flows, where different types of flow are co-located in the same route or occur at the same time, we can more accurately manage, both spatially and temporally, the synergies of multiple travel flows of residents visiting urban parks. Previous studies have identified spatial bundles of ecosystem services using correlation coefficient analysis in many cities [24], while temporal bundles of ecosystem services are seldom identified. Studies on travel behavior have identified distinct travel groups using cluster analysis based on individual travel paths or socio-economic and demographic characteristics to represent homogenous groups [25]. However, as travel flows of residents visiting urban parks have received limited attention, their bundles, therefore, have rarely studied, either in space or time.

Although spatial-temporal characteristics of activity-based travel flows have been quantified, few studies have attempted to focus on the characteristics of travel flows of residents visiting urban parks. This study seeks to fill this research gap by mapping and quantifying spatial and temporal dynamics and bundles of travel flows in Wuhan, P.R. China. The study was based on empirical investigation of individuals' travel movements from their homes to the parks. The research was guided by the 
following two research questions: How are travel flows of residents visiting urban parks spatially and temporally distributed in the study area, and what groups of bundled travel flows can emerge in relation to space and time constraints?

\section{Methods}

\subsection{Data Collection}

\subsubsection{Park Sampling}

This study was conducted in the center of Wuhan, P.R. China. At the end of 2016, 33 free public parks were unevenly distributed across seven urban districts within an area of $863 \mathrm{~km}^{2}$ in the central part of the city. To exclude dilapidated and rarely used parks, we first selected a sample of 21 main public parks that receive more than 100,000 visitors per year, according to data from the Wuhan Municipal Bureau of Landscape and Forestry. We divided these 21 parks into three size classes: small ( $<10 \mathrm{ha})$, medium (10-50 ha), and large parks ( $>50 \mathrm{ha})$. We then randomly selected one park from each size category in each urban district, to ensure that our analysis covered different categories of park size and all the city's central areas. Finally, a total of 12 public parks were selected, representing $57 \%$ of the 21 main public parks (including $67 \%$ of the small parks, $56 \%$ of the medium parks, and $50 \%$ of the large parks) in the study area. Figure 1 shows their locations.

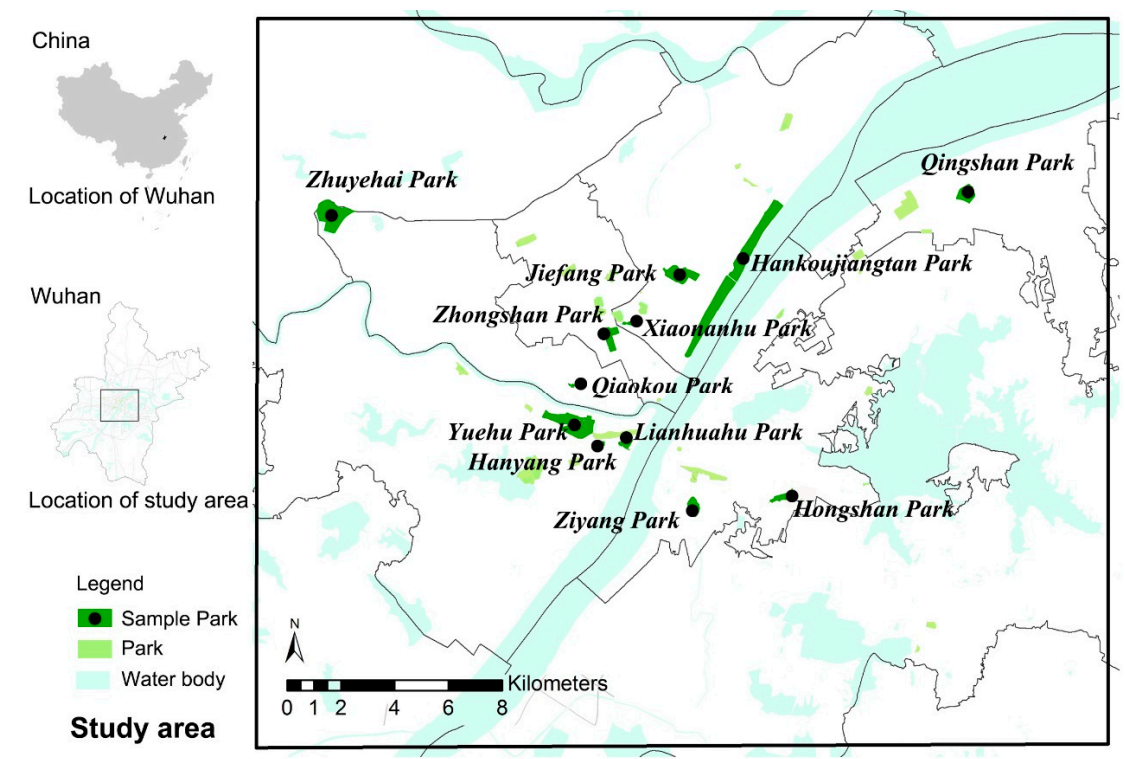

Figure 1. Study area and location of parks studied in Wuhan.

\subsubsection{Questionnaire}

To understand the travel behavior of people from their homes to the parks, face-to-face questionnaire surveys were conducted in the 12 selected parks during the summers of 2015 and 2016. Visitors passing research assistants or resting in places that were not too busy at the entrance inside the park were invited, at random, to participate in the investigation. However, only local visitors between 14 and 75 years old were included in the survey. The on-site interviews started with an introduction and clarification of the purpose of the study, and written informed consent was obtained from each participant before the questionnaire survey. We used a one-page questionnaire, which was discussed with an expert panel (the authors and five professors in relevant disciplines) in an effort to ensure it had sufficient face validity. A pre-investigation involving 50 respondents was conducted in Zhongshan Park to test the clarity of the questionnaire prior to conducting the formal survey. The final questionnaire was divided into two main sections. The first covered how visitors travel to the parks, 
including questions on their reasons for travel and sequence of movements, where they live, which park entrance they used, what times they arrive and leave the park, which mode of transport they use, and how often they visit. To comply with privacy policies, respondents were asked to write the location of the nearest bus stop to their residence instead of their home address. The second section of the questionnaire was designed to elicit information on respondents' demographic characteristics, including their age, gender and occupation.

\subsection{Mapping and Quantifying Spatial Travel Flows}

The daily park travel flows of individuals reflect the spatial connections between urban parks and residents. Individual travel choices and movements are widely recognized to have significant effect on their travel flow patterns [17], including travel flow paths, distances, velocities, quantities, and frequencies. Flow path (in this context) refers to the travel trajectory, from the location of an individual's residence to the park entrance they used, while flow distance is the length of the flow path. The movement of people can be characterized by their travel velocity and frequency, which are determined by their choice of mode of transport and frequency of park visits, respectively. Flow quantity refers to the number of residents travelling to parks, and often declines with increasing travel distance. These dimensions are integrated together to form individual descriptions of travel flows.

The process of data selection was conducted before the mapping analysis. Only travel episodes directly from home to the park were considered. To estimate each respondent's flow path, the shortest path along the road network connecting the park entrances they used to the bus stop closest to their residence was extracted using Baidu Map (a popular online search engine that Chinese urban residents use extensively for daily navigation), together with their reported mode of transport. Then, all extracted paths were converted into line shape file format in a geographical information system (GIS), and mode of transport, frequency, park arrival and departure time attributes were assigned to each of these lines. As described in most previous studies, mode of transport and frequency determine the inherent characteristics of travel flows $[13,16]$. Mode of transport choice reflects different movement velocities and affects the experience of the trip for park use. Travel frequency is considered in terms of travel for recreational purposes, indicating individuals' preferences. To further distinguish travel flows with inherent differences, we classified the flows into nine permutations of low, medium and high frequencies and velocities, thus separating the flows into groups that shared similar characteristics, as shown in the matrix presented in Table 1. According to the questionnaire survey, visitors have four direct transport options for reaching urban parks: walking, driving, catching a bus, or taking the metro. We classified walking as the low velocity mode, and taking the metro as the high velocity mode, while driving and catching a bus were classified as the medium velocity mode, based on their relative speeds. According to common perceptions of residents in Wuhan, visiting a park more than three times per week ( $>150$ times/year) was classified as high frequency, and visiting less than once a week ( $<50$ times/year) was considered low frequency, with intermediate values classified as medium frequency. This classification system was selected because it is consistent with a common understanding and is easy both to communicate and understand. Next, nine corresponding sets of line shape files were extracted from the converted path files, mentioned above, using ArcGIS software.

To map spatial flows of travel to parks, a density surface of each flow path type was generated by kernel density estimation (KDE) analysis using the line shape file in ArcGIS. The KDE method has been widely used to describe spatial distributions of densities (of diverse variables) with distance by smoothing data converted into continuous density surfaces [26]. In KDE analysis, a kernel's bandwidth determines the accuracy of results [27]. In total, six bandwidths were tested with the same output cell size $(10 \mathrm{~m} \times 10 \mathrm{~m})$ and same kernel function in this study: 20, 50, 100, 200, 300, and $400 \mathrm{~m}$. The inflexion point of the relationship between bandwidth and the maximum KDE value was used to estimate the optimal bandwidth for KDE analysis, and local hotspots of mapped events (here, flows) were displayed for verification. An output cell size of $10 \mathrm{~m} \times 10 \mathrm{~m}$ was used because it provided sufficient precision for distinguishing individuals' travel behavior from closed travel routes according 
to the spacing between adjacent roads in the study area. Finally, a kernel bandwidth of $100 \mathrm{~m}$ was employed, selected on the basis of the tests mentioned above. A flow chart of the procedure is shown in Figure 2.

Descriptive statistics were used to characterize different types of travel flows, including length of flow path, travel time and frequency of park visits. For each variable, both the mean and standard deviation (SD) were calculated using SPSS software.

Table 1. Classification of travel flows.

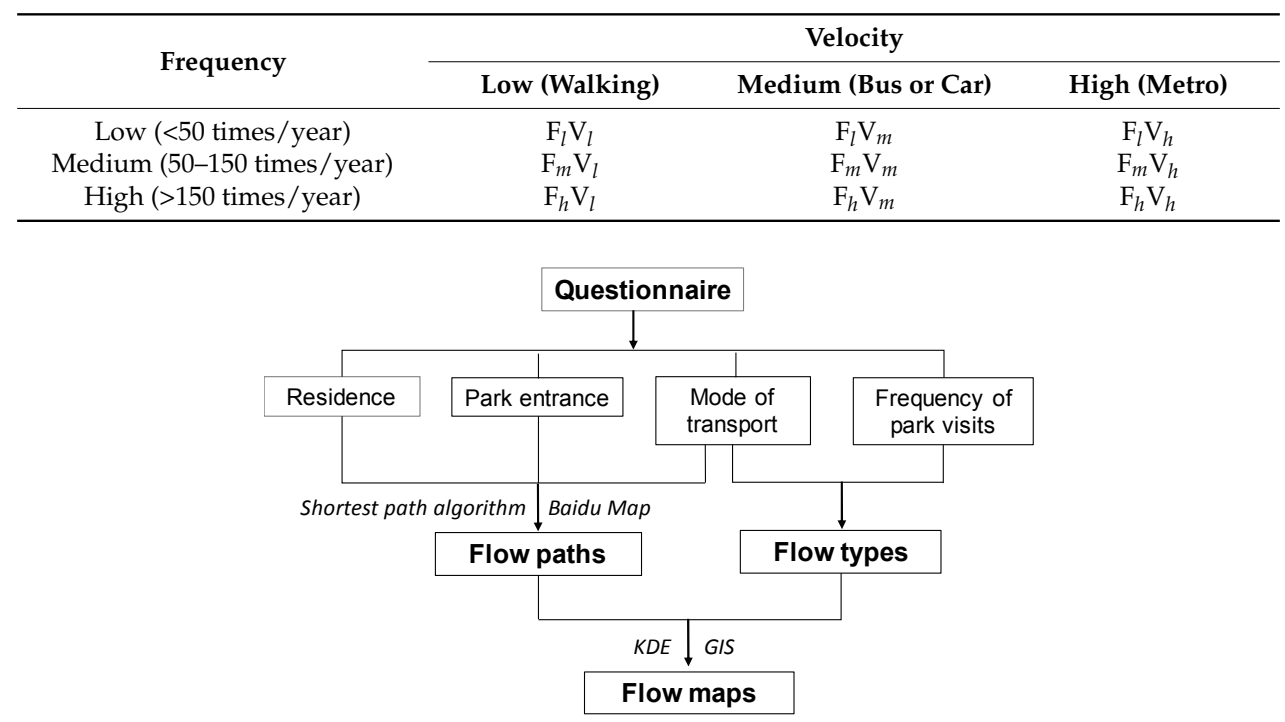

Figure 2. Flow chart showing the procedure for mapping travel flows of residents visiting parks.

\subsection{Identifying Temporal Dynamics of Travel Flows}

To identify daily patterns of movement, we counted numbers of the respondents arriving and leaving in the questionnaire in one-hour intervals separately, based on the questionnaire survey. Scatter plots were then constructed to display the distributions of numbers of park visitors, and optimal fitting models (Peak, Gaussian, three parameters) were used to describe the temporal patterns for each category of travel flow. To illustrate the differences in patterns between arrival and departure times, the scatter plots and regression lines of the times were mapped together in the same figure for each category of flow. All these analyses were conducted using Sigmaplot 12.0 software.

\subsection{Bundling Spatial and Temporal Travel Flows}

As different types of travel flow may have similar spatial and temporal distributions, the coexistence of different types of flow with respect to space and time must be examined. This is achieved by applying the concept of "bundles" of travel flows that share similar characteristics. To explore spatial bundles of flows, we first converted road network lines generated by all travel paths into location points at $100 \mathrm{~m}$ intervals. Then, KDE values for each of the nine types of travel flow were extracted from the point shape files, thus nine KDE values were separately assigned to the attributes of each location point. These spatial location points were then used as samples for hierarchical cluster analysis to identify spatial bundles of travel flows, using the nine types of KDE values for each point as the cluster field. We ran agglomerative hierarchical cluster analysis using Euclidian distance and standardization of values to Z scores in SPSS 19.0 software. The hierarchical tree generated by this unification process was plotted to present a visual representation of the bundled groups of spatial flows.

Cluster and Outlier Analysis was used to estimate temporal bundles of travel flow paths that shared similar service times, using the Anselin Local Moran I statistic with inverse distance weighting 
in the ArcGIS environment. This procedure generates statistically significant clusters of high values (High-High), clusters of low values (Low-Low), outliers with high values surrounded by low values (High-Low), and outliers with low values surrounded by high values (Low-High). Here, lines of the flow paths were input as the analysis objects, while arrival times were used as the analysis field. Thus, High-High clusters, Low-Low clusters, High-Low outliers and Low-High outliers can be respectively regarded as flow bundles with late arrival times, flow bundles of with early arrival times, isolated flow paths of with late arrival times, and isolated flow paths of with early arrival times.

\section{Results}

\subsection{Respondents' Profiles}

In total, we conducted 4904 direct face-to-face interviews, which provided validly completed questionnaires from 4854 participants (2754 males and 2100 females). Just $6.12 \%$ of respondents were under 18 years old, but the age of adult respondents was well balanced: 31.33, 28.6 and $33.9 \%$ were $18-40,40-60$ and $>60$ years old, respectively. Nearly half the respondents were retired (46.9\%), $20.9 \%$ were formal employment jobs, $21.1 \%$ were freelancers, and $11.1 \%$ were students.

\subsection{Characteristics of Spatial Travel Flows}

The spatial distributions of the nine types of travel flow to the urban parks are presented in Figure 3. The black areas on the maps represent high flows, indicating large movements of people from residences to parks, while the blank areas represent no flows. Clearly, travel flows of residents visiting urban parks were not randomly distributed in the study area. Low velocity flows were strongly clustered around the park destinations (Figure $3 \mathrm{a}, \mathrm{d}, \mathrm{g}$ ), and lengths of these flow paths were inversely related to frequency.

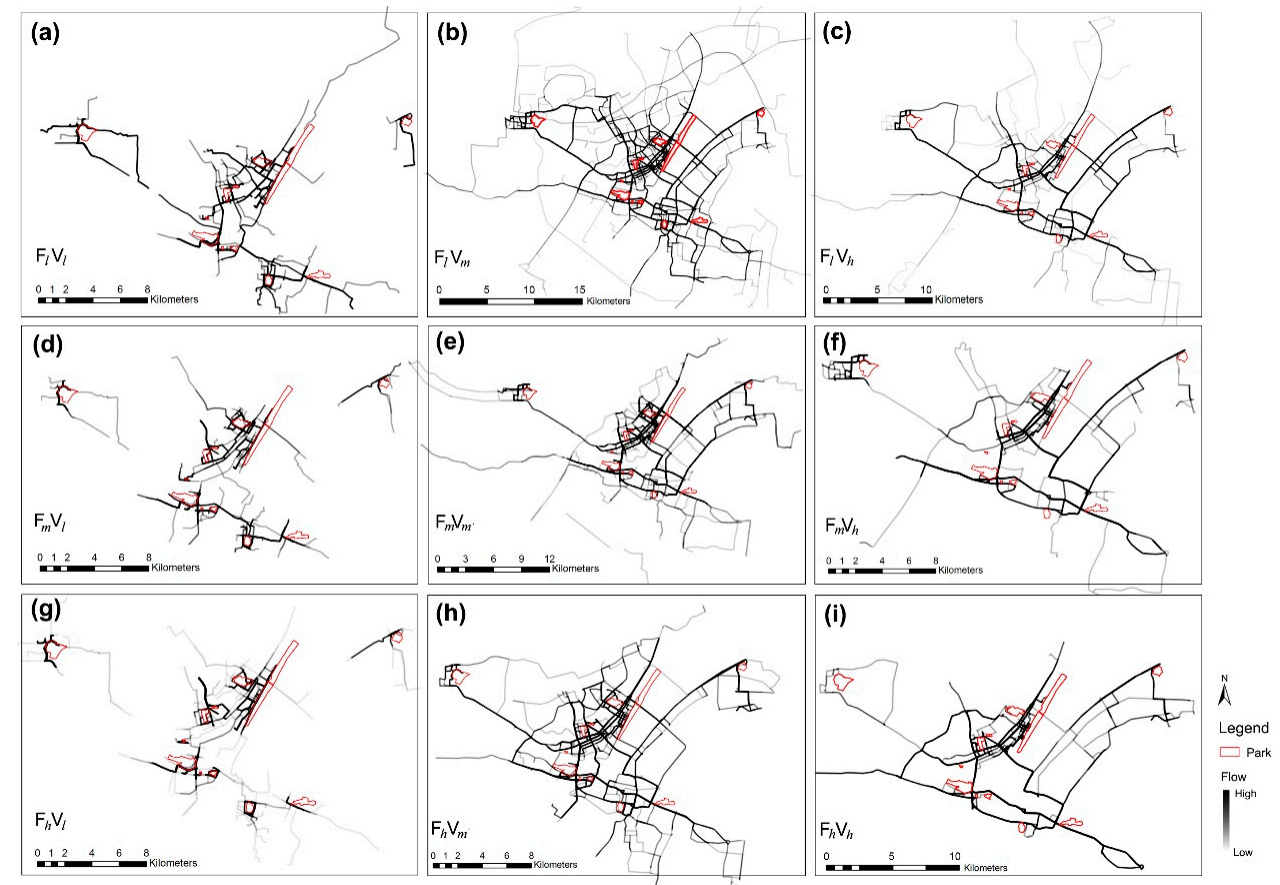

Figure 3. KDE features of travel flows with: (a) low frequency and low velocity; (b) low frequency and medium velocity; (c) low frequency and high velocity; (d) medium frequency and low velocity; (e) medium frequency and median velocity; (f) medium frequency and high velocity; (g) high frequency and low velocity; (h) high frequency and medium velocity; (i) high frequency and high velocity. $\mathrm{F}$ and $\mathrm{V}$ refer to frequency and velocity, respectively, while the superscripts $l, m$ and $h$ refer to low, medium and high, respectively. 
For instance, paths of flows with low velocity and high frequency were the shortest, predominantly at the periphery of the park (Figure 3g). In contrast, high velocity flows were mainly associated with long distances, but path densities of these flows were often low (Figure $3 \mathrm{c}, \mathrm{f}, \mathrm{i})$. Moreover, flows with high velocity and low frequency were extensively distributed across the whole study area (Figure 3c). Medium velocity flows were associated with high flow path densities and long travel distances (Figure 3b,e,h).

Table 2 shows results of the statistical analysis of the nine types of travel flow. Different types of flow with the same velocity had similar average flow path lengths and travel times. The average flow path length and travel time of low velocity flows was $<2 \mathrm{~km}$ and approximately $20 \mathrm{~min}$, respectively. These constituted most of the flows (64.5\%) in the study area, indicating that most urban parks are located in walking distance of the visitors' homes. Low velocity-high frequency flows accounted for the highest proportion (40.9\%), and had the highest frequency (320 times per year on average). About a quarter of park user's travel by car or bus to reach the parks, representing the medium velocity flows. Average lengths of these journeys were between $5 \mathrm{~km}$ and $9 \mathrm{~km}$, and their average travel time was about half an hour. Average lengths of the high velocity flows exceeded $9 \mathrm{~km}$, and their average travel time exceeded half an hour. Proportions of these flows were very low in the study area $(8.9 \%)$, especially those with high velocity and high frequency $(1.2 \%)$.

Table 2. Characteristics of types of travel flow. F and V refer to frequency and velocity, respectively, while the superscripts $l, m$ and $h$ refer to low, medium and high, respectively.

\begin{tabular}{|c|c|c|c|c|c|c|c|}
\hline \multirow{2}{*}{ Type } & \multicolumn{2}{|c|}{ Length of Flow Routes (km) } & \multicolumn{2}{|c|}{ Travel Time (min) } & \multicolumn{2}{|c|}{ Frequency (No. Year ${ }^{-1}$ ) } & \multirow{2}{*}{ N (\%) } \\
\hline & Mean & SD & Mean & SD & Mean & SD & \\
\hline $\mathrm{F}_{l} \mathrm{~V}_{l}$ & 1.87 & 2.41 & 20.3 & 18.4 & 22 & 17 & 11.02 \\
\hline $\mathrm{F}_{l} \mathrm{~V}_{m}$ & 8.94 & 7.88 & 34.8 & 24.4 & 17 & 16 & 15.35 \\
\hline $\mathrm{F}_{l} \mathrm{~V}_{h}$ & 13.63 & 7.13 & 47.9 & 20.7 & 14 & 14 & 6.20 \\
\hline $\mathrm{F}_{m} \mathrm{~V}_{l}$ & 1.53 & 1.54 & 15.6 & 13.9 & 104 & 30 & 12.57 \\
\hline $\mathrm{F}_{m} \mathrm{~V}_{m}$ & 5.69 & 6.05 & 26.6 & 21.1 & 92 & 32 & 5.95 \\
\hline $\mathrm{F}_{m} \mathrm{~V}_{h}$ & 9.15 & 4.93 & 36.6 & 15.2 & 91 & 25 & 1.59 \\
\hline $\mathrm{F}_{h} \mathrm{~V}_{l}$ & 1.59 & 1.39 & 19.1 & 16.6 & 320 & 66 & 40.94 \\
\hline $\mathrm{F}_{h} \mathrm{~V}_{m}$ & 5.38 & 4.52 & 28.0 & 18.9 & 285 & 80 & 5.23 \\
\hline $\mathrm{F}_{h} \mathrm{~V}_{h}$ & 9.99 & 5.70 & 38.3 & 17.7 & 280 & 83 & 1.15 \\
\hline
\end{tabular}

\subsection{Temporal Dynamics of Travel Flows}

The scatter plots and regression lines displayed in Figure 4 represent the temporal patterns of respondents' movements from their homes to the parks for the nine categories of travel flow $(p<0.0001)$. Obviously, residents in Wuhan prefer to visit parks in the morning. Departure times were about two to three hours after arrival times, indicating that most of the respondents spent $2-3 \mathrm{~h}$ in the park. During the morning peak, most types of travel flow started during the period 8:00-9:00 a.m. and ended in the period 11:00-12:00 a.m. (Figure 4). Generally, both the arrival and departure times associated with high frequency flows were earlier than those for low frequency flows. For instance, peak arrival and departure times for high frequency-high velocity flows were approximately 8:00 am and 10:00 a.m., respectively (Figure 4i), while corresponding times for low frequency-high velocity flows were approximately 9:00 am and 12:00 a.m., respectively (Figure 4c). Medium frequency-high velocity flows had the smallest ranges of arrival (8:00-10:00) and departure times (10:00-14:00) (Figure 4f), while high frequency-high velocity flows had the largest range of arrival times (4:00-12:00) (Figure 4i), and low frequency-low velocity flows had the largest range of departure times (7:00-15:00) (Figure 4a). There were several small visiting peaks in the afternoon, at 12:00-14:00 and 16:00-18:00, or evening (at 20:00) for several types of travel flows, although numbers of visitors during these peaks were relatively low. 

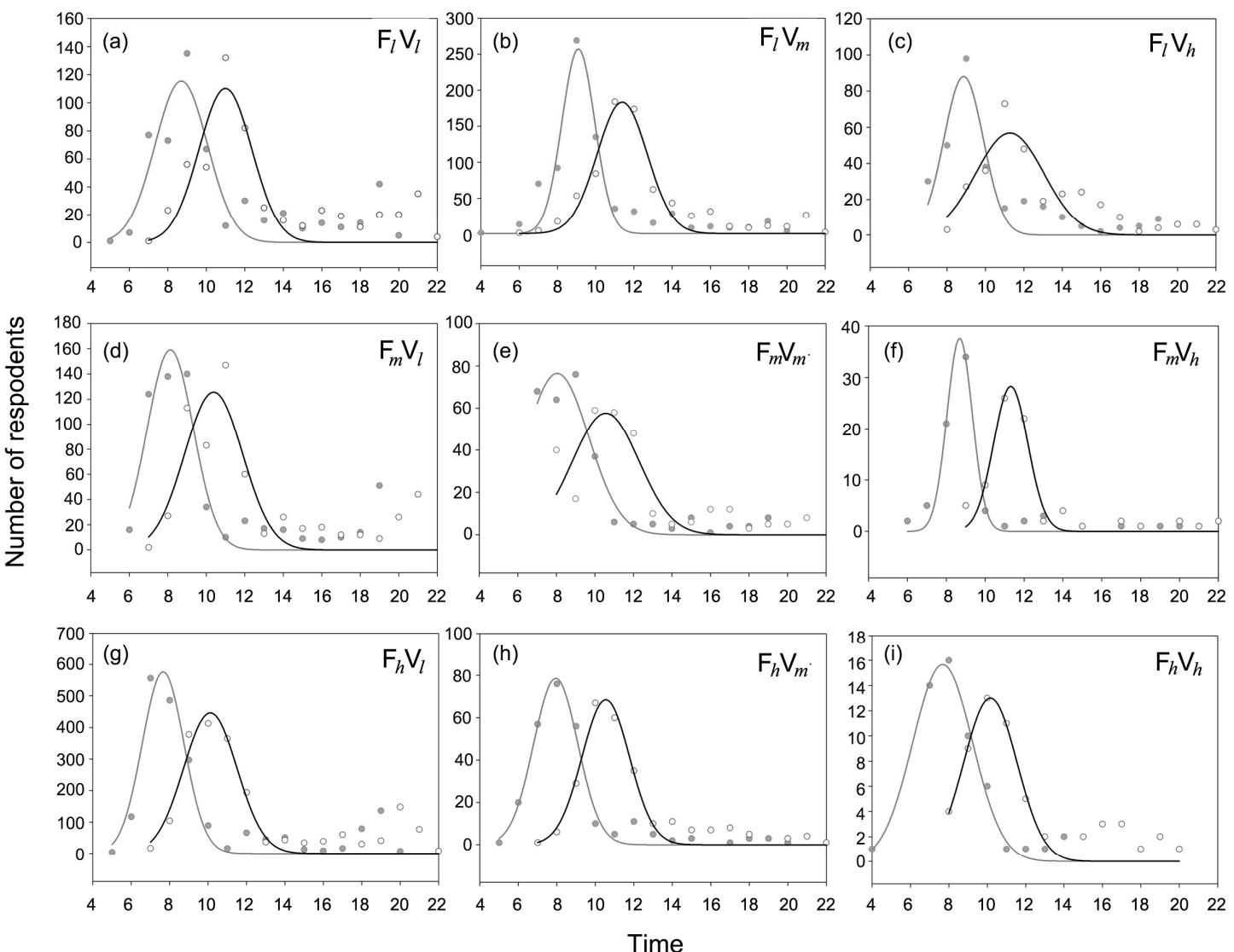

- Arrival time $\quad$ Departure time

Arrival time \& Number of respodents

Departure time \& Number of respodents

Figure 4. Distribution over time of travel flows with: (a) low frequency and low velocity; (b) low frequency and medium velocity; (c) low frequency and high velocity; (d) medium frequency and low velocity; (e) medium frequency and medium velocity; (f) medium frequency and high velocity; (g) high frequency and low velocity; (h) high frequency and medium velocity; (i) high frequency and high velocity. F and $\mathrm{V}$ refer to frequency and velocity, respectively, while the superscripts $l, m$ and $h$ refer to low, medium and high, respectively.

\subsection{Spatial and Temporal Bundles of Travel Flows}

Results of hierarchical cluster analysis showed that the nine types of travel flow of respondents visiting urban parks could be grouped into four clusters at a similarity level of approximately 5 (Figure 5), representing four spatial bundles of travel flows that share similar spatial locations. The first bundle included all three high velocity flows, indicating that park visitors travelling to parks by metro may choose the same routes no matter how often they visit the parks. The second bundle included the flows with medium velocity and high or low frequency, while the third bundle consisted of medium frequency-medium velocity flows, suggesting that residents who visit parks two to three times per week by car or bus shared similar travel routes, and other residents visiting parks by car or bus take another paths. The fourth bundle included low velocity flows of all frequencies, which implies that frequent or non-frequent visitors may choose similar walkable routes to parks.

Figure 6 shows results of the cluster and outlier analysis, which reveals associations (at a 0.05 probability level) between four significant bundles of flow paths from homes to the parks and the associated arrival times. High-High clusters were distributed across the entire study area, notably showing strong connections between the southeast and northwest. Low-Low clusters were mainly aggregated in the densely populated regions of the city center with a high density of flow paths, 
indicating that residents who live in the city center have more route choices to access parks in the early morning. The results also showed that there were several High-Low outliers distributed in the south and east of the study area, while Low-High outliers mainly occurred in the sparsely populated northwest of the study area. However, it should be noted that fewer paths belong to both Low-High and High-Low outliers compared to the other clusters. This means that residents who visit parks at different times have limited route choices. Flows with no statistically significant clusters were mainly distributed in the north of the study area, indicating the non-repetitive distributed travel flows with respect to arriving time at the park.

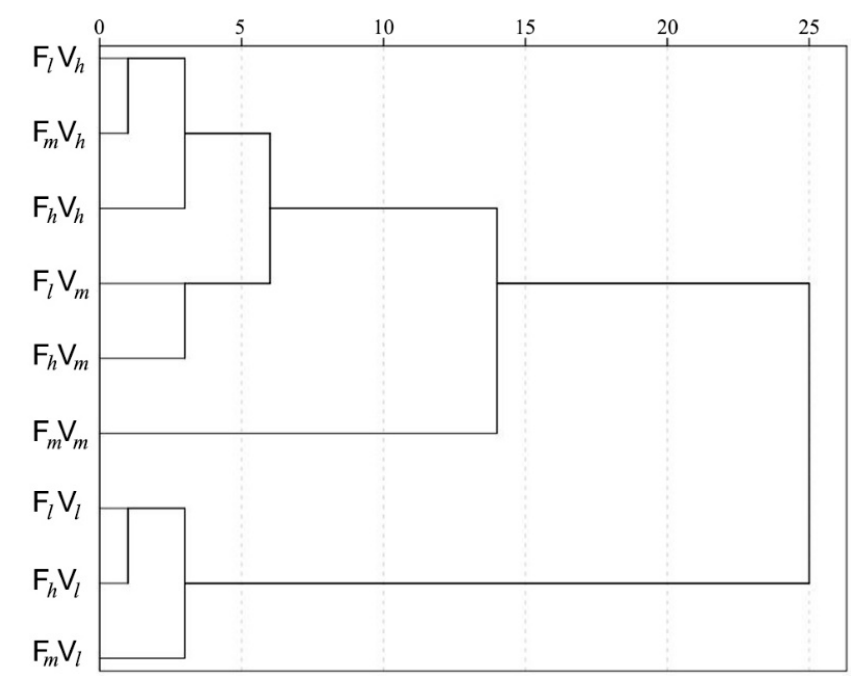

Figure 5. Dendrogram obtained from the hierarchical cluster analysis, showing four main clusters at a similarity of approximately 5 (dotted line), representing four spatial bundles of travel flows. F and V refer to frequency and velocity, respectively, while the superscripts $1, \mathrm{~m}$ and $\mathrm{h}$ refer to low, medium and high, respectively.

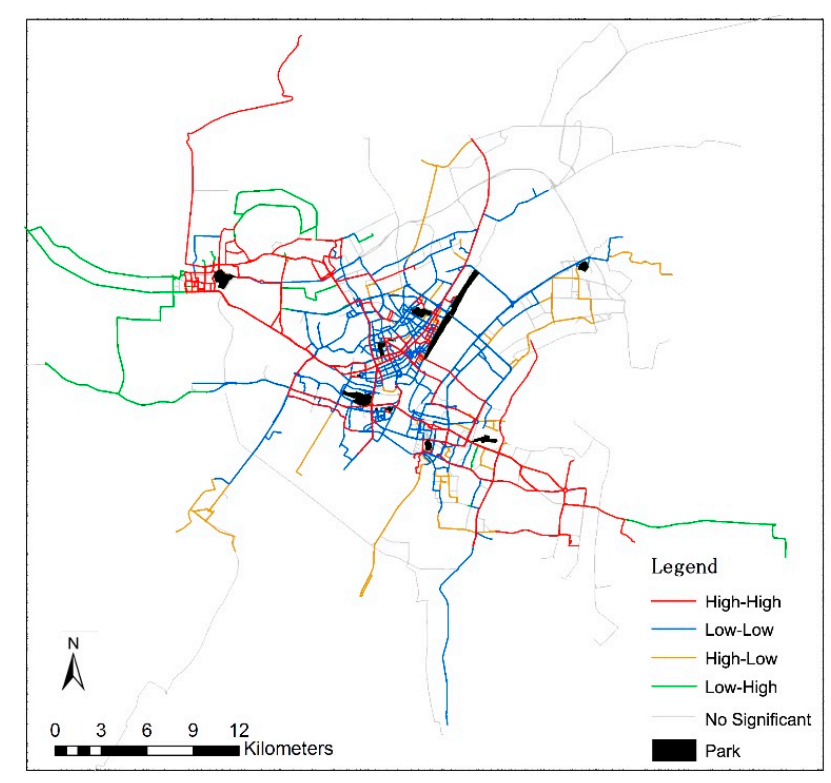

Figure 6. Results of Cluster-Outlier analysis of travel flows' associations with arrival times, representing temporal bundles of the flows. High-High (clusters), Low-Low (clusters), High-Low (outliers) and Low-High (outliers) can be, respectively, regarded as flow bundles with late arrival times, flow bundles with early arrival times, isolated flow paths with late arrival times, and isolated flow paths with early arrival times. 


\section{Discussion}

\subsection{Travel Flows for Visiting Parks}

We have expanded previous approaches for quantifying travel flows of residents visiting urban parks by combining estimated densities of travel paths with respondents' self-reported modes of transport, based on questionnaire survey and online map navigation technology. The flow maps of travel to visit parks generated in our study capture key flow patterns showing where, which, and how much of the travel flows were being undertaken with the purpose of using the recreational services of urban parks. This provides new insights into residents' preferences towards different transportation options. With such flow maps, decision-makers or spatial planners could easily identify park access inequity, and plan interventions more precisely to restore or improve the efficiency of park use; for example, adjusting park distribution, or optimizing transit networks. On the other hand, combining urban land use classification or other functional zones with these flow maps, the supply of public facilities and services (their quantity, size and location) could be correspondingly adjusted for a synthesis public service use.

Our results show that the travel flows of residents visiting urban parks were not scattered randomly, and a large proportion of low velocity flows were distributed around the parks in the form of short, low-density paths (especially high frequency-low velocity flows). This is probably because most frequent visitors walk to nearby parks, but pedestrian-friendly roads and footpaths are not extensively distributed around the parks in the study area. Increasing networks of such routes around the parks might be necessary to increase travel by food. In contrast, few paths were associated with high frequency-high velocity flows, although most of them were long distance paths. This might be because quick modes of transport (metro) enable park visitors to travel long distances over a relatively short period of time, eliminating the inconveniences of road transport, but distant visitors do not often access parks for various park-based or user-based reasons [28-30]. For example, people who live far away may not visit small parks often, while older visitors may prefer to visit nearby parks [31]. It is also notable that the flows of medium velocity were extensively distributed across the whole study area, with high densities of flow paths, indicating that visitors from many directions travel to parks by bus or car. The main reason for these residents to travel by car is that many of the parks considered are not well connected with the residents within a walkable distance. However, as these flows only account for approximately a quarter of the total, and with increasing private car ownership in Wuhan, increasing the service efficiency of the car and bus routes appears to be necessary. In addition, few flows identified in this study were located in the east of the study area, where lakes and steep hills cover large areas (see Appendix A), so the road density and park accessibility are low. A growing population in this area, associated with rapid urban development in Wuhan, is likely to increase demands for park recreational services. If so, the spatial constraints will pose major challenges to improving travel efficiency and balancing the supply and demand of park recreational services in the east. Further research is needed to address this issue.

Our results highlight the temporal patterns of people's regular movements to the parks, and reveal that few visitors (especially frequent visitors) access and use parks at midday or in the afternoon. This temporal feature of travel flows is probably related to the high midday and afternoon temperatures and humidity in summer in Wuhan [32,33], known as one of China's "furnace cities". Correspondingly, cooler temperatures might explain the visiting peaks for all flows in the morning. Besides temperature, the small evening visiting peaks of several types of flow may be because office workers spend some of their leisure time after work in the parks. However, several types of flow (with very low numbers) showed a small visiting peak in the afternoon. Most of these few afternoon park visits appeared to be brief, indicating that it was a matter of the opportunity. Overall, travel to urban parks is essentially time constrained, and managers should be aware that it is important for opening hours of urban parks to coincide with the times that visitors find it convenient to travel. Estimates of temporal 
distributions of travel flows and peak park visiting hours could help city planners to improve service time management, and thus the service use efficiency of urban parks.

\subsection{Bundles of Trips to Parks}

The main variable distinguishing bundles of spatial travel flows for park visits identified in this study was flow velocity, indicating that transport conditions are the main factors affecting the bundling of spatial flows rather than frequency of park visits (at least in Wuhan during the summer study periods). This finding suggests that integrating flows with different velocities into the same paths may require spatial trade-offs. Such trade-offs are often necessary to optimize the balance between travel experience and service delivery efficiency. For example, encouraging walking as an independent mode of transport for visiting parks could be beneficial in terms of travel safety and comfort, but the movement of people using rapid modes of transport requires other paths, and hence demands more roads for service delivery. However, integrating flows with different velocities in the same paths may be necessary due to urban space limitations; this approach can be generally employed for routes used by road-based modes of transport. Panter et al. [34] reported that some people were more likely to combine walking and cycling with their car journeys when the route environment is supportive, allowing flows with different velocities in single paths. At the aggregate level, such bundles represent a dramatic reduction in variations in travel to visit the parks, thus allowing spatial planners to observe the main flow distribution and trends of residents travelling to parks. However, this is a challenging option in cities with poorly established traffic systems, although a cautious policy combining road-based visitors in the same routes could improve transport efficiency and ensure more park visitor flow across the area in a limited urban space. Conversely, our investigation indicates that it is highly possible to integrate flows with different frequencies in the same path to enhance service delivery; this provides an insight into travel routes that are common to visitors of different frequencies. Such insights into trade-offs and synergies of different types of flow in different urban contexts could be helpful for improving the efficiency of travelling to urban parks.

Another clear finding is that the temporal patterns of clusters of travel flows were significantly related to spatial factors, which is consistent with the findings of most studies that human movement is regular, both spatially and temporally $[4,7,35]$. This finding makes it possible to manage residents' travel routes based on their routine travel to parks. Most of the flows with early arrival times in our study were associated with short travel distances, indicating that they are mainly generated by the movement of people residing close to the parks. Flows with long travel distances were clustered as bundles with late arrival times, possibly for similar reasons. Given the importance of avoiding traffic jams at particular times, these flows with different travel distances may be related to trade-offs with arrival time. Developing a practical time management system based on the temporal bundles of travel flow maps, would be valuable for helping to avoid overcrowding when residents travel to parks. Conversely, results of the travel flow outliers indicated use of the same flow paths with different arrival times, possibly because there are few traffic jams in the suburbs, so people have a range of choices of times to visit the parks via the same route. These findings suggest that service time management is another potentially important means to enhance efficiency of recreational use of urban parks.

\subsection{Limitations and Future Research}

In this study, individuals' movement paths from their homes to park destinations were estimated using the shortest path algorithm together with their self-reported mode of transport. However, sometimes park visitors may not actually follow the shortest path [21]. For example, travelers often take detours to avoid traffic jams, or because they have incomplete information about transport options and conditions [35]. Some authors have also found that visitors select travel routes based on travel time rather than the shortest path $[21,36]$. It is difficult to identify specific flow paths from the limited data provided by questionnaires. Recently, the rise of big data has provided opportunities to elucidate individual's actual travel behavior at an operational level. Hence, for instance, some studies have tried 
to visualize and quantify travel paths based on GPS trajectories of visitors [14], individual's mobile phones [37] and smart card data [15]. However, individuals' travel purposes cannot yet be readily extracted from such sources, so they have not yet been used to analyze mobility patterns from homes to parks. Thus, combining traditional data sources and data obtained from new technology is a major challenge, but essential to refine the mapping and quantification of travel flows of visitors to urban parks in further research.

Travelling to urban parks, is fact, not only related to space and time constraints of travel routes, but also spatial-physical characteristics of the parks and socio-demographic factors [38], such as available park facilities, population density around the parks, visitors' gender and household structure [5,35]. Understanding such physical and non-physical drivers may be important for addressing critical travel flows for recreational use of urban parks. Hence, interactions between space and time constraints and socio-demographic factors and their impact on the management of dynamic travel flows for recreational use of urban parks should be further investigated in future studies.

\section{Conclusions}

Quantifying travel flows of residents visiting urban parks, with respect to space and time, is important for understanding the processes involved in, and factors affecting, the recreational use of urban parks. This paper presents a visual impression of the spatial delivery and temporal dynamics of park visitor flows in Wuhan in summer, by mapping the movements of residents from their homes to parks, based on empirical investigation. The flow maps could help efforts to characterize park service efficiency and identify social inequities in park access from a user's standpoint. Analysis of the temporal dynamics of travel flows showed that distributions of park visits are significantly time constrained. Thus, temporal dynamics of park visits should be considered in urban park management, especially peak arrival and departure times, which may help in the development of more dynamic strategies to meet different residents' park visiting needs. Moreover, the analysis of bundles of travel flows from spatial and temporal perspectives, suggests that transport conditions strongly influence bundling of the spatial flows, whilst bundling of temporal flows is significantly related to spatial factors. These findings may help efforts to identify potential trade-offs and synergies among different types of travel flow, and facilitate exploration of policy alternatives to provide equitable and efficient urban park systems.

Acknowledgments: This work was funded by the Natural Science Foundation of China (51508218) and the Fundamental Research Funds for the Central Universities (2662015QC025, 2662015BQ010).

Author Contributions: Wenping Liu designed the study and contributed to the analyzing and writing the paper; Weijuan Chen and Chenlu Dong collected and processed the data. All authors have read and approved the final manuscript.

Conflicts of Interest: The authors declare no conflict of interest. 


\section{Appendix A}

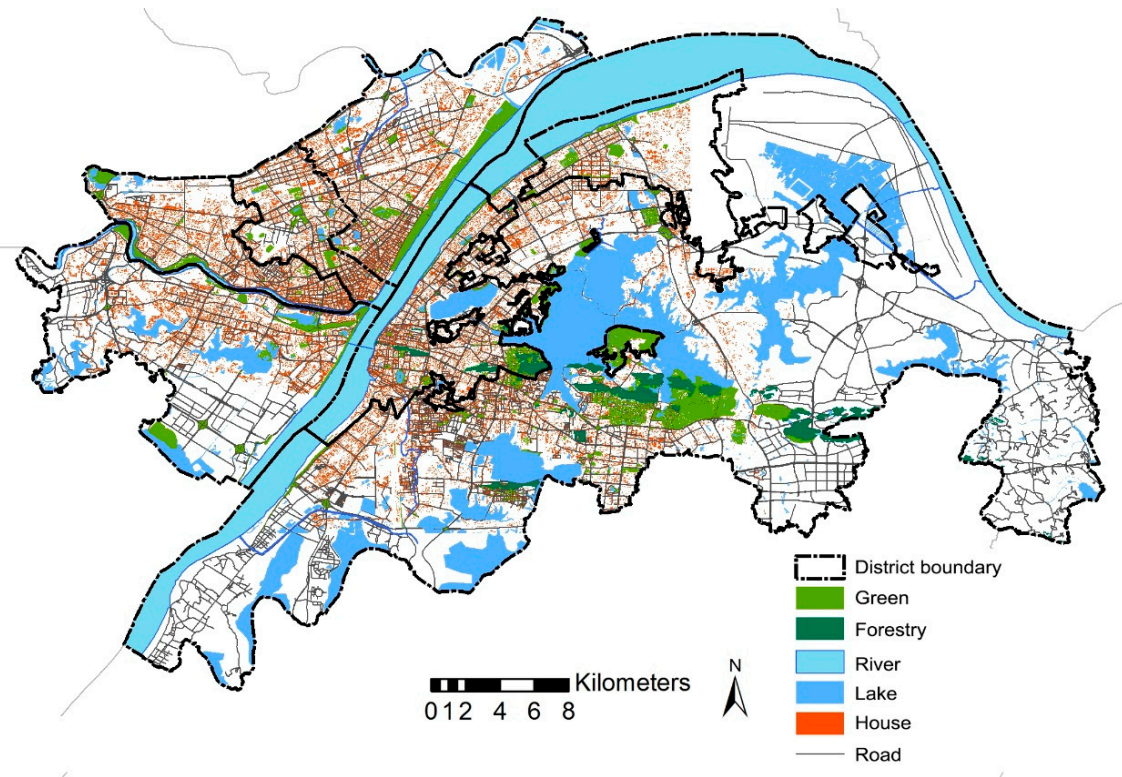

Figure A1. Land use map in the central urban area of Wuhan.

\section{References}

1. Brown, G.; Schebella, M.F.; Weber, D. Using participatory GIS to measure physical activity and urban park benefits. Landsc. Urban Plan. 2014, 121, 34-44. [CrossRef]

2. Dzhambov, A.M.; Dimitrova, D.D. Elderly visitors of an urban park, health anxiety and individual awareness of nature experiences. Urban For. Urban Green. 2014, 13, 806-813. [CrossRef]

3. Vrebos, D.; Staes, J.; Vandenbroucke, T.; Johnston, R.; Muhumuza, M.; Kasabeke, C.; Meire, P. Mapping ecosystem service flows with land cover scoring maps for data-scarce regions. Ecosyst. Serv. 2015, 13, $28-40$. [CrossRef]

4. Buliung, R.N.; Roorda, M.J.; Remmel, T.K. Exploring spatial variety in patterns of activity-travel behaviour: Initial results from the Toronto Travel-Activity Panel Survey (TTAPS). Transportation 2008, 35, 697-722. [CrossRef]

5. Feng, J.; Dijst, M.; Wissink, B.; Prillwitz, J. The impacts of household structure on the travel behaviour of seniors and young parents in China. J. Transp. Geogr. 2013, 30, 117-126. [CrossRef]

6. Roorda, M.J.; Ruiz, T.S. Long- and short-term dynamics in activity scheduling: A structural equations approach. Transp. Res. Part A Policy Pract. 2008, 42, 545-562. [CrossRef]

7. Thøgersen, J. Understanding repetitive travel mode choices in a stable context: A panel study approach. Transp. Res. Part A Policy Pract. 2006, 40, 621-638. [CrossRef]

8. Taff, D.; Newman, P.; Pettebone, D.; White, D.D.; Lawson, S.R.; Monz, C.; Vagias, W.M. Dimensions of alternative transportation experience in Yosemite and Rocky Mountain National Parks. J. Transp. Geogr. 2013, 30, 37-46. [CrossRef]

9. Mace, B.L.; Marquit, J.D.; Bates, S.C. Visitor Assessment of the Mandatory Alternative Transportation System at Zion National Park. Environ. Manag. 2013, 52, 1271-1285. [CrossRef] [PubMed]

10. Bertram, C.; Meyerhoff, J.; Rehdanz, K.; Wüstemann, H. Differences in the recreational value of urban parks between weekdays and weekends: A discrete choice analysis. Landsc. Urban Plan. 2017, 159, 5-14. [CrossRef]

11. Hewer, M.; Scott, D.; Fenech, A. Seasonal weather sensitivity, temperature thresholds, and climate change impacts for park visitation. Tour. Geogr. 2016, 18, 297-321. [CrossRef]

12. Czajkowski, M.; Ahtiainen, H.; Artell, J.; Budziński, W.; Hasler, B.; Hasselström, L.; Meyerhoff, J.; Nõmmann, T.; Semeniene, D.; Söderqvist, T.; et al. Valuing the commons: An international study on the recreational benefits of the Baltic Sea. J. Environ. Manag. 2015, 156, 209-217. [CrossRef] [PubMed] 
13. Orsi, F.; Geneletti, D. Assessing the effects of access policies on travel mode choices in an Alpine tourist destination. J. Transp. Geogr. 2014, 39, 21-35. [CrossRef]

14. Zheng, W.; Huang, X.; Li, Y. Understanding the tourist mobility using GPS: Where is the next place? Tour. Manag. 2017, 59, 267-280. [CrossRef]

15. Yu, C.; He, Z. Analysing the spatial-temporal characteristics of bus travel demand using the heat map. J. Transp. Geogr. 2017, 58, 247-255. [CrossRef]

16. Smallwood, C.B.; Beckley, L.E.; Moore, S.A. An analysis of visitor movement patterns using travel networks in a large marine park, north-western Australia. Tour. Manag. 2011, 33, 517-528. [CrossRef]

17. Orellana, D.; Bregt, A.K.; Ligtenberg, A.; Wachowicz, M. Exploring visitor movement patterns in natural recreational areas. Tour. Manag. 2012, 33, 672-682. [CrossRef]

18. Bagstad, K.J.; Johnson, G.W.; Voigt, B.; Villa, F. Spatial dynamics of ecosystem service flows: A comprehensive approach to quantifying actual services. Ecosyst. Serv. 2013, 4, 117-125. [CrossRef]

19. Johnson, G.; Bagstad, K.; Snapp, R.; Villa, F. Service Path Attribution Networks (SPANs): A Network Flow Approach to Ecosystem Service Assessment. Int. J. Agric. Environ. Inf. Syst. 2012, 3, 54-71. [CrossRef]

20. Byon, Y.J.; Abdulhai, B.; Shalaby, A. Incorporating Scenic View, Slope, and Crime Rate into Route Choices. Transp. Res. Rec. 2010, 2183, 91-102. [CrossRef]

21. Alivand, M.; Hochmair, H.; Srinivasan, S. Analyzing how travelers choose scenic routes using route choice models. Comput. Environ. Urban Syst. 2015, 50, 41-52. [CrossRef]

22. Zheng, Y.; Yan, S.; Zha, Z.; Li, Y.; Zhou, X.; Chua, T.; Jain, R. GPSView: A scenic driving route planner. ACM Trans. Multimedia Comput. Commun. Appl. 2013, 9, 3. [CrossRef]

23. Susilo, Y.; Dijst, M. How Far Is Too Far? Travel time ratios for activity participations in the Netherlands. Transp. Res. Rec. 2009, 2134, 89-98. [CrossRef]

24. Raudsepp-Hearne, C.; Peterson, G.D.; Bennett, E.M. Ecosystem service bundles for analyzing tradeoffs in diverse landscapes. Proc. Natl. Acad. Sci. USA 2010, 107, 5242-5247. [CrossRef] [PubMed]

25. Lin, H.; Lo, H.; Chen, X. Lifestyle classifications with and without activity-travel patterns. Transp. Res. Part A Policy Pract. 2009, 43, 626-638. [CrossRef]

26. Maroko, A.R.; Maantay, J.A.; Sohler, N.L.; Grady, K.L.; Arno, P.S. The complexities of measuring access to parks and physical activity sites in New York City: A quantitative and qualitative approach. Int. J. Health Geogr. 2009, 8, 34. [CrossRef] [PubMed]

27. Xie, Z.; Yan, J. Kernel Density Estimation of traffic accidents in a network space. Comput. Environ. Urban Syst. 2008, 32, 396-406. [CrossRef]

28. Giles-Corti, B.; Broomhall, M.H.; Knuiman, M.; Collins, C.; Douglas, K.; Ng, K.; Lange, A.; Donovan, R.J. Increasing walking: How important is distance to, attractiveness, and size of public open space? Am. J. Prev. Med. 2005, 28, 169-176. [CrossRef] [PubMed]

29. Jones, A.; Hillsdon, M.; Coombes, E. Greenspace access, use, and physical activity: Understanding the effects of area deprivation. Prev. Med. 2009, 49, 500-505. [CrossRef] [PubMed]

30. Shan, X. The socio-demographic and spatial dynamics of green space use in Guangzhou, China. Appl. Geogr. 2014, 51, 26-34. [CrossRef]

31. Liu, W.; Chen, W.; Dong, C. Spatial decay of recreational services of urban parks: Characteristics and influencing factors. Urban For. Urban Green. 2017, 25, 130-138. [CrossRef]

32. Lin, T.; Tsai, K.; Hwang, R.; Matzarakis, A. Quantification of the effect of thermal indices and sky view factor on park attendance. Landsc. Urban Plan. 2012, 107, 137-146. [CrossRef]

33. Xu, X.; Ge, Q.; He, S.; Zhang, X.; Xu, X.; Liu, G. Impact of high temperature on the mortality in summer of Wuhan, China. Environ. Earth Sci. 2016, 75, 543. [CrossRef]

34. Panter, J.; Desousa, C.; Ogilvie, D. Incorporating walking or cycling into car journeys to and from work: The role of individual, workplace and environmental characteristics. Prev. Med. 2013, 56, 211-217. [CrossRef] [PubMed]

35. Ta, N.; Zhao, Y.; Chai, Y. Built environment, peak hours and route choice efficiency: An investigation of commuting efficiency using GPS data. J. Transp. Geogr. 2016, 57, 161-170. [CrossRef]

36. Bierlaire, M.; Frejinger, E. Route choice modeling with network-free data. Transp. Res. Part C Emerg. Technol. 2008, 16, 187-198. [CrossRef] 
37. Phithakkitnukoon, S.; Horanont, T.; Witayangkurn, A.; Siri, R.; Sekimoto, Y.; Shibasaki, R. Understanding tourist behavior using large-scale mobile sensing approach: A case study of mobile phone users in Japan. Pervasive Mob. Comput. 2015, 18, 18-39. [CrossRef]

38. Plieninger, T.; Dijks, S.; Oteros-Rozas, E.; Bieling, C. Assessing, mapping, and quantifying cultural ecosystem services at community level. Land Use Policy 2013, 33, 118-129. [CrossRef] 